\title{
The association between antihypertensive drugs and glioma
}

\author{
MPWA Houben, ${ }^{*}, 2$, JWW Coebergh ${ }^{2,3}$, RMC Herings $^{4}$, MK Casparie ${ }^{5}$, CC Tijssen', CM van Duijn ${ }^{2}$ and \\ BHCh Stricker ${ }^{2}$ \\ 'Department of Neurology, St Elisabeth Hospital, PO Box 90151, 5000 LC Tilburg, The Netherlands; ${ }^{2}$ Department of Epidemiology \& Biostatistics, \\ Erasmus MC, Rotterdam, The Netherlands; ${ }^{3}$ Comprehensive Cancer Centre South, Eindhoven, The Netherlands; ${ }^{4}$ Pharmo Institute, Utrecht, \\ The Netherlands; ${ }^{5}$ Foundation PALGA, Utrecht, The Netherlands
}

We pursued an association between hypertension and gliomas by investigating whether antihypertensive drugs (AHD) are associated with an increased glioma risk by a population-based nested case-control study using the PHARMO database; this links dispensing records of prescription drugs to hospital discharge data on an individual basis. Pathological data were derived from the Dutch nationwide registry of histo- and cytopathology. A total of 306 glioma cases incident between 1997 and 2003 were matched to I I 08 controls for year of birth, sex, geographical region and duration of follow-up. Exposure was defined as cumulative duration of AHD use and, in an alternative analysis, as cumulative dose. We estimated the magnitude of the association with conditional logistic regression analysis. Cumulative use of any AHD for more than 6 months was associated with an increased risk of glioma (OR I.45: 95\% Cl 1.03-2.04). After stratification for different groups of AHD, no significantly increased risk of glioma was found for any class of AHD. After excluding a latency period of 3 years before the date of diagnosis, no association was found. In conclusion, the use of AHD seems to be associated with an increased risk of glioma, but this is probably not causal.

British Journal of Cancer (2006) 94, 752-756. doi: I 0.1038/sj.bjc.6603000 www.bjcancer.com

Published online 2I February 2006

(c) 2006 Cancer Research UK

Keywords: glioma; risk; antihypertensive drugs; pharmaco-epidemiology; case-control study

Although gliomas are the most common type of primary brain tumour, they are nevertheless relatively rare. In the Netherlands, world-standardised incidence rates of glioma are 6.5 per 100000 person-years for male and 4.4 for female subjects (van der Sanden et al, 1998). The aetiology of glioma remains largely unclear and ionising radiation is the only established environmental risk factor, but the exposure rate is low (Ron et al, 1988; Karlsson et al, 1998). Some rare genetic disorders exist in which patients have an increased risk of glioma and a variety of other cancers (Louis and von Deimling, 1995; Melean et al, 2004).

Previously, we found a significantly higher prevalence of hypertension in glioma patients in a population-based case-control study in the Eindhoven Cancer Registry (Houben et al, 2004). We hypothesised that one possible mechanism for this association is through potentially neurocarcinogenic effects of antihypertensive drugs (AHD) or their metabolites. To test the hypothesis of a glioma-inducing effect of AHD, we conducted a population-based nested case-control study with prospectively collected automated pharmacy data, linked to morbidity and pathology data.

\section{MATERIALS AND METHODS}

\section{Setting}

We used the PHARMO record linkage system, a database that since 1985 has linked dispensing records of prescription drugs from a

*Correspondence: Dr MPWA Houben, Department of Neurology, St Elisabeth Hospital, PO Box 90 I5I, 5000 LC Tilburg, The Netherlands; E-mail: m.houben@erasmusmc.nl

Received 24 November 2005; revised 12 January 2006; accepted 18 January 2006; published online 2I February 2006 representative sample of Dutch community pharmacies to hospital discharge data on an individual basis (Herings, 1993). These pharmacies are scattered over the country and currently cover data of more than two million residents, equivalent to $12 \%$ of the Dutch population. Participants enter the database when their first prescription is filled in a PHARMO community pharmacy, and they are followed thereafter. The recorded information of each drug includes the Anatomical Therapeutic Chemical (ATC) code (WHO, 1990), the dispensing date, the quantity of the drug, the prescribed daily dose, and the estimated duration of use. Because almost all persons designate a single pharmacy to fill their drug prescriptions, dispensing histories are virtually complete (Lau et al, 1997).

Drug dispensing histories are linked to hospital discharge records from the Dutch Medical Register (LMR), using a validated and reliable probabilistic algorithm (Herings, 1993). The LMR comprises all hospital admissions in the Netherlands and includes dates of admission and discharge, discharge diagnoses, comorbidity and performed medical procedures. All diagnoses are coded according to the International Classification of Diseases, ninth edition clinical modification (ICD-9-CM). Since 1991, the primary diagnosis is determined by the specialist who treated the patient. Other sources of morbidity and drug exposure include hospital pharmacies, clinical laboratories and general practitioners.

\section{Study population and validation}

As glioma is not a separate code in the ICD-9-CM, all patients with a brain tumour (ICD-9-CM code 191) in the LMR database were linked to the Dutch nationwide network and registry of histo- and cytopathology (PALGA) containing data of histological, cytological and autopsy examinations of all 16 million inhabitants in the 
Netherlands. We included all incident gliomas from 1 January 1997 to 31 December 2003 to include at least 12 years of potential medication histories since 1985 . The index date was defined as the first admission date for a brain tumour, which was (later) confirmed to be a glioma by clinical pathology. All patients with a previous history of nonglioma cancer before the index date were excluded, identified by a history of cancer (ICD-9-CM codes 140208), a history of radiotherapy or use of anticancer medications (ATC codes L01, L02 and L03). For each case, we took 3-4 controls from the PHARMO database, matched on geographical region, date of birth (5-year intervals), gender and duration of follow-up in the PHARMO database (3-month intervals). Duration of followup was defined as the difference between the date of entry in PHARMO and the index date. Controls were assigned the same index date as the cases and were part of the PHARMO population on this date. To be certain that controls were free of (yet undetected) cancer at the index date, controls with a history of cancer up to 2 years after the index date were excluded. A history of cancer in controls was identified as in the cases.

\section{Exposure definition}

In the Netherlands, all AHD are prescription-only drugs. For all cases and controls, the complete history of filled prescriptions before the index date was obtained. The following AHD were analysed: ATC codes C02 (miscellaneous AHD), C03 (diuretics), C07 (beta-blockers), C08 (calcium antagonists) and C09 (ACE inhibitors and ATII antagonists). Exposure was calculated as the duration of use before the index date in number of days, and as the sum of dispensed defined daily doses (DDD). The DDD is the recommended standard dose for the main indication in adults, as defined by the World Health Organisation. As it is highly unlikely that occasional short-term use causes glioma, we defined a prior cutoff point of cumulative use of 6 months.

\section{Statistical analysis}

We restricted the analyses to patients aged 30 years and over. We assessed ever use of AHD before the index date as a potential risk factor for glioma. We compared patients and controls with respect to cumulative duration of use and cumulative dose. Diuretics, beta-blockers, calcium antagonists, drugs acting on the reninangiotensin system (ACE inhibitors, ATII antagonists) and miscellaneous AHD were analysed separately, adjusted for the other ATC classes. We also stratified for gender, age and histological subgroups of glioma.

To exclude the possibility that use of AHD was started because of symptoms in the prodromal phase of glioma, and to take into account the delay between tumour induction and diagnosis, we performed sensitivity analyses by subtracting a lag time of 3 years from the index date as performed before (Beiderbeck et al, 2003). $\chi^{2}$ statistics were used to compare proportions. Conditional logistic regression was used to estimate the association between AHD and the risk of glioma, expressed as odds ratios (ORs) and 95\% confidence intervals (CI). Analyses were adjusted for residual variation in age and duration of follow-up. Since the cumulative

Table I Characteristics of glioma cases and controls

\begin{tabular}{|c|c|c|c|c|c|}
\hline \multirow[b]{2}{*}{ Characteristic } & \multicolumn{2}{|c|}{ Patients $(n=306)$} & \multicolumn{2}{|c|}{ Controls $(n=1108)$} & \multirow[b]{2}{*}{$P$-value } \\
\hline & $n$ & $\%^{\mathbf{a}}$ & $n$ & $\%^{\mathbf{a}}$ & \\
\hline \multicolumn{6}{|l|}{ Age (years) } \\
\hline $30-44$ & 66 & 21.6 & 227 & 20.5 & \\
\hline $45-59$ & 115 & 37.6 & 440 & 39.7 & \\
\hline $60-74$ & 100 & 32.7 & 363 & 32.8 & \\
\hline$\geqslant 75$ & 25 & 8.2 & 78 & 7.0 & 0.84 \\
\hline \multicolumn{6}{|l|}{ Sex } \\
\hline Men & 184 & 60.1 & 669 & 60.4 & \\
\hline Women & 122 & 39.9 & 439 & 39.6 & 0.94 \\
\hline \multicolumn{6}{|l|}{ Follow-up time in PHARMO (years) } \\
\hline 0 to $<1$ & 43 & 14.1 & 164 & 14.8 & \\
\hline I to $<3$ & 53 & 17.3 & 204 & 18.4 & \\
\hline Median, 25th-75th percentile & $5.4,2.1-10.1$ & & $5.2,2.0-10.1$ & & \\
\hline \multicolumn{6}{|l|}{ Diagnosis } \\
\hline Astrocytoma, glioblastoma multiforme & 252 & 82.3 & & & \\
\hline Oligodendroglioma & 32 & 10.5 & & & \\
\hline Ependymoma & 3 & 1.0 & & & \\
\hline Mixed glioma & 15 & 4.9 & & & \\
\hline Glioma not otherwise specified & 4 & 1.3 & & & \\
\hline \multicolumn{6}{|l|}{ Cumulative duration of AHD use } \\
\hline No use & 197 & 64.4 & 804 & 72.6 & \\
\hline$<6$ months & 35 & 11.4 & 95 & 8.6 & \\
\hline 6 months to 4 years & 40 & 13.1 & 98 & 8.8 & \\
\hline
\end{tabular}

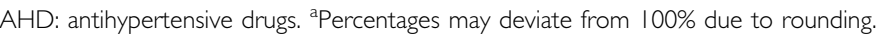


dose can be the result of prolonged use of low-dose AHD, or of short-term high-dose AHD, we also adjusted for duration of use in the analyses for cumulative dose. In the analyses for cumulative duration of use, we additionally adjusted for mean DDD per day of use. All statistical tests were performed two-sided and statistical significance was indicated if $P<0.05$. Analyses were performed using SPSS for Windows version 12.0.1 and Statistical Analysis System version 8.2 .

\section{RESULTS}

The baseline characteristics of 306 glioma patients and 1108 matched controls aged over 30 years are shown in Table 1 . The most frequent subtype was astrocytic glioma (82.3\%), followed by oligodendroglioma $(10.5 \%)$ and mixed glioma $(4.9 \%)$, this distribution and the male excess $(60.1 \%)$ being comparable with those previously reported (van der Sanden et al, 1998; Kleihues and Cavenee, 2000). The median follow-up time in PHARMO was 5.4 years for patients and 5.2 years for controls. The cumulative duration of AHD use was longer in patients than in controls $(P=0.03)$.

In the conditional logistic regression analyses, an increased risk of glioma was found for users of any AHD (OR 1.45; 95\% CI $1.03-$ 2.04) (Table 2). When exposure was measured as cumulative dose, a nonsignificantly increased risk was found for users who received $>180$ DDD compared with users who received $<180$ DDD (OR 1.35; $95 \%$ CI $0.86-2.11$ ). After stratification for ATC classes, none of the other risk estimates reached statistical significance, although numbers were small in some of the exposure categories (Table 2). The risk of glioma was elevated for users of miscellaneous AHD (OR 1.96; 95\% CI 0.71-5.37), but not significantly.

Comparable risks were seen when exposure was measured as cumulative dose (not shown). Again, a nonsignificantly increased risk was found for users of miscellaneous AHD (OR 2.05; 95\% CI $0.65-6.49)$. These results did not change when adjusted for the mean DDD per day of use (not shown).
In the sensitivity analyses, subtracting 3 years from the index date moved the risk estimates toward unity (Table 3), and no increased risk for users of any AHD could be shown (OR 1.08; 95\% CI $0.69-1.70$ ).

We also stratified for diagnosis (astrocytoma only, oligodendroglioma only), gender and age, and analysed for the use of any AHD. This did not change the results (not shown). In particular, no association was found for elderly men, who have the highest prevalence of hypertension (Houben et al, 2004).

\section{DISCUSSION}

This is the first detailed study of the effect of AHD use on glioma risk, using prospectively collected automated pharmacy data. The higher risk of glioma for users of any AHD disappeared when a lag time of 3 years was applied; none of the different classes of AHD showed statistically significant associations.

An association between AHD-use and cancer risk is not established. There is evidence that renal carcinoma is associated with diuretic use (Grossman et al, 2001), although this is not generally accepted (Choi et al, 2005; Fryzek et al, 2005). Prenatal exposure to diuretics was associated with an increased risk of childhood brain tumours (Preston-Martin et al, 1982), which could not be confirmed by subsequent studies (Kuijten et al, 1990; McCredie et al, 1994; McKean-Cowdin et al, 2003). The risks of exposure to AHD in adulthood have not been thoroughly investigated although no increased risk could be shown for diuretic use (Ryan et al, 1992). No association was found between blood pressure and brain tumours, but numbers of cases were small, the effect of AHD was not investigated, and gliomas were not studied as a separate group (Batty et al, 2003).

We previously found a higher prevalence of hypertension in glioma patients (Houben et al, 2004). Since glioma is not known to induce clinically relevant hypertension and because hypertension is not a known risk factor for glioma, we hypothesised that the use of AHD might be the link in the observed association. The results

Table 2 Associations between the duration of use of antihypertensive drugs and glioma, without considering a lag period of exposure

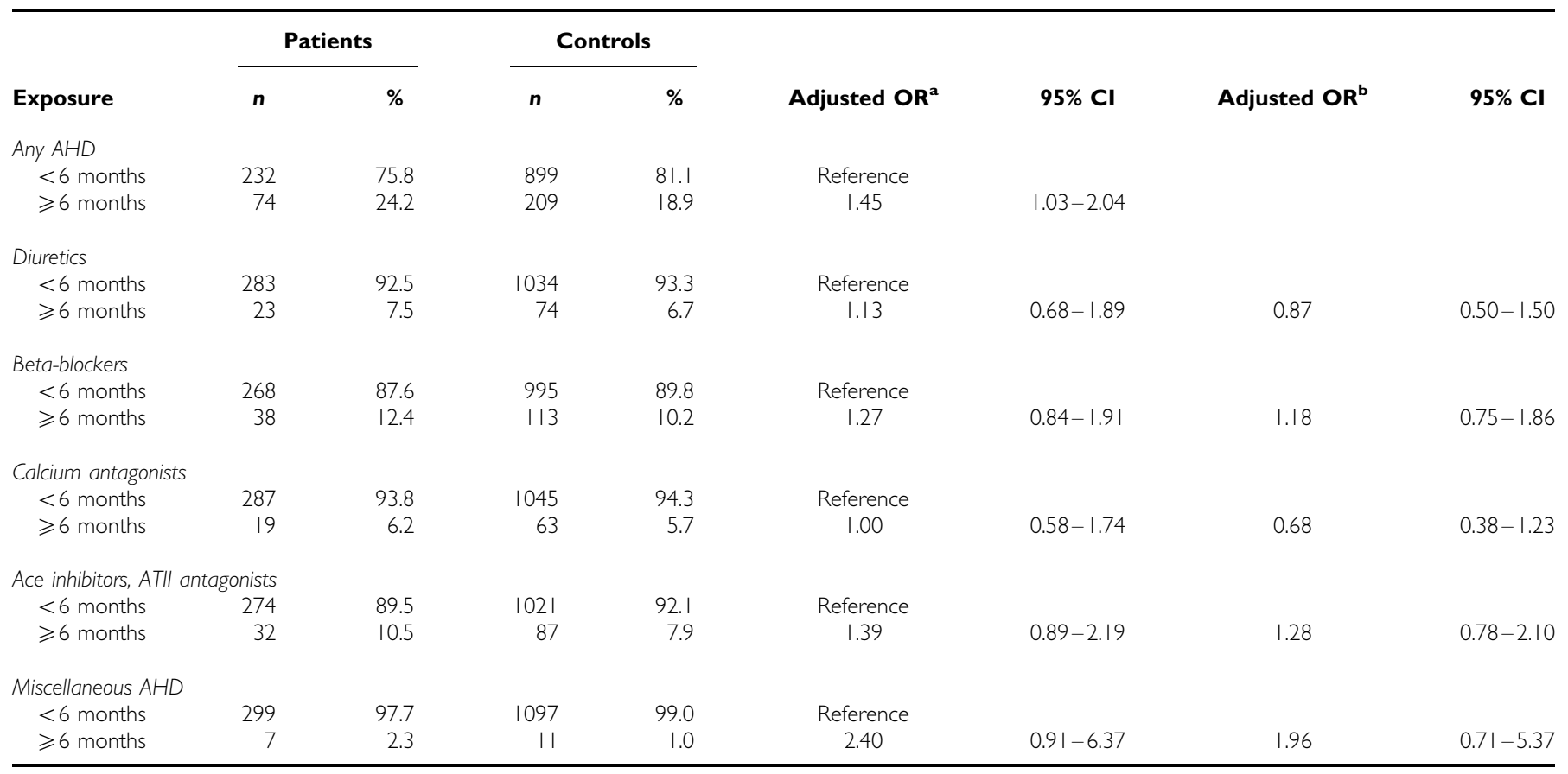

AHD: antihypertensive drugs; OR: odds ratio; Cl: confidence interval. ${ }^{a}$ Adjusted for age, gender and duration of follow-up. ${ }^{b}$ Adjusted for age, gender, duration of follow-up and for use of other types of antihypertensive drugs (ever vs never use). 
Table 3 Associations between the duration of use of antihypertensive drugs and glioma, considering a lag period of exposure of 3 years

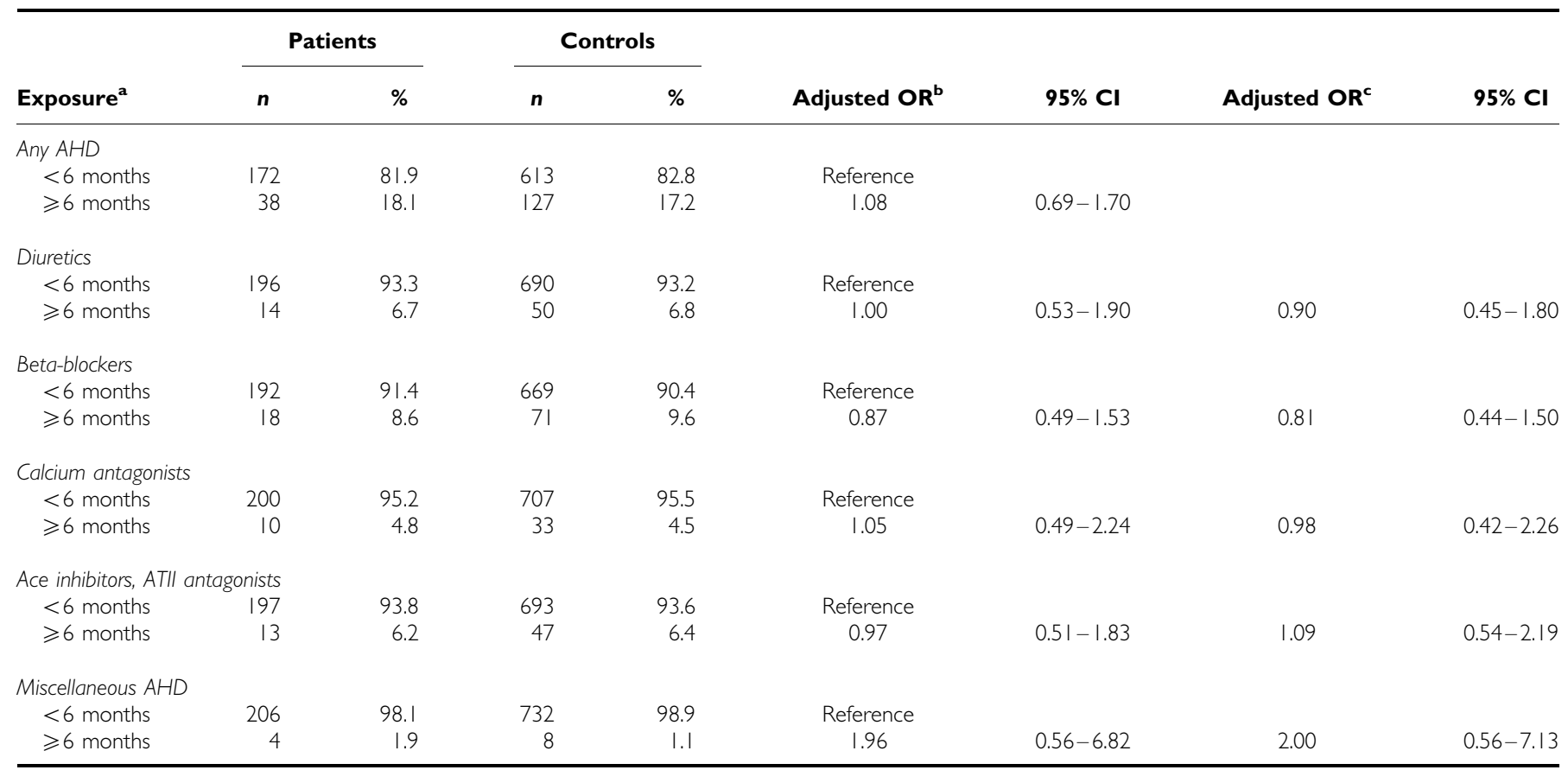

AHD: antihypertensive drugs; OR: odds ratio; Cl: confidence interval. ${ }^{\mathrm{A}} \mathrm{A}$ lag period of exposure was considered by subtracting 3 years from the index date. ${ }^{\mathrm{b}} \mathrm{Adjusted}$ for age, gender and duration of follow-up. 'Adjusted for age, gender, duration of follow-up and for use of other types of antihypertensive drugs (ever vs never use).

of this study do not support such an association. Notably, potential associations disappeared in the sensitivity analyses, suggesting that hypertension is part of the prodromal signs of glioma prompting prescription of AHD though it must be conceded that such a presentation of glioma is not recognised.

Major strengths of this study are its population-base and its use of prospectively collected data on drug exposure and pathology. Pharmacy records are more complete and more reliable than medical records or patient interviews, thereby avoiding recall bias (Paganini-Hill and Ross, 1982). It has been shown that computerised pharmacy records are a reliable source of true current drug exposure (Lau et al, 1997), and that any misclassification is nondifferential, leading to underestimation of the true effect, rather than the reverse, in pharmaco-epidemiological studies (Lau et al, 1997). We considered information about glioma diagnosis to be reliable since several sources were combined, including PALGA, which is not only used for research purposes but for daily patient care. Detection bias seems unlikely as gliomas will almost always become symptomatic, regardless of medical surveillance. We therefore did not adjust for the effect of medical attention due to comorbidity, but did adjust for gender, age, duration of follow-up and geographical region. Because the PHARMO database does not contain information on lifestyle variables, we were not able to adjust for obesity, smoking or alcohol use. However, we do not expect this to be a problem since none of these factors has been associated with glioma (Wrensch et al, 2002).

\section{REFERENCES}

Batty GD, Shipley MJ, Marmot MG, Davey Smith G (2003) Blood pressure and site-specific cancer mortality: evidence from the original Whitehall Study. Br J Cancer 89: $1243-1247$

Beiderbeck AB, Holly EA, Sturkenboom MC, Coebergh JW, Stricker BH, Leufkens HG (2003) Prescription medications associated with a decreased risk of non-Hodgkin's lymphoma. Am J Epidemiol 157: $510-516$
A potential problem is that the duration of observation might have been too short to study the influence of AHD on glioma risk, possibly leading to an underestimation of risk. Furthermore, we studied five categories of AHD, based on the main mode of action. Within these categories, however, drugs can have different modes of action. For instance, ACE inhibitors and angiotensin II inhibitors are both classified in ATC group C09, but might differ in their ability to induce or promote glioma. The numbers of cases were too small to analyse these AHD separately. Similarly, for the group of miscellaneous AHD, numbers of cases were too small to draw conclusions.

In conclusion, a causal association between AHD and glioma seems unlikely, although we cannot exclude the possibility that AHD modify the occurrence of glioma. Both overall exposure and exposure to subgroups of AHD could not be clearly related to a higher risk of glioma. Some associations disappeared in the sensitivity analyses, indicating that hypertension might be a prodromal sign of glioma.

\section{ACKNOWLEDGEMENTS}

We are grateful to the staff of the PHARMO Institute and PALGA who were very helpful in the data selection procedures. Financial support was provided by the Dutch Cancer Society, Grant EUR 2001-2454.

Choi MY, Jee SH, Sull JW, Nam CM (2005) The effect of hypertension on the risk for kidney cancer in Korean men. Kidney Int 67: 647-652

Fryzek JP, Poulsen AH, Johnsen SP, McLaughlin JK, Sorensen HT, Friis S (2005) A cohort study of antihypertensive treatments and risk of renal cell cancer. $\mathrm{Br}$ J Cancer 92: $1302-1306$

Grossman E, Messerli FH, Goldbourt U (2001) Antihypertensive therapy and the risk of malignancies. Eur Heart J 22: 1343-1352 
Herings R (1993) Pharmo, A Record Linkage System for Postmarketing Surveillance of Prescription Drugs in The Netherlands (Dissertation). The Netherlands: Utrecht University

Houben MPWA, Louwman WJ, Tijssen CC, Teepen JLJM, Van Duijn CM, Coebergh JWW (2004) Hypertension as a risk factor for glioma? Evidence from a population-based study of comorbidity in glioma patients. Ann Oncol 15: 1256-1260

Karlsson P, Holmberg E, Lundell M, Mattsson A, Holm LE, Wallgren A (1998) Intracranial tumors after exposure to ionizing radiation during infancy: a pooled analysis of two Swedish cohorts of 28008 infants with skin hemangioma. Radiat Res 150: 357-364

Kleihues P, Cavenee WK (2000) Pathology \& Genetics of Tumours of the Central Nervous System. World Health Organization Classification of Tumours. Lyon, France: IARC Press

Kuijten RR, Bunin GR, Nass CC, Meadows AT (1990) Gestational and familial risk factors for childhood astrocytoma: results of a case-control study. Cancer Res 50: 2608-2612

Lau HS, de Boer A, Beuning KS, Porsius A (1997) Validation of pharmacy records in drug exposure assessment. J Clin Epidemiol 50: 619-625

Louis DN, von Deimling A (1995) Hereditary tumor syndromes of the nervous system: overview and rare syndromes. Brain Pathol 5: $145-151$

McCredie M, Maisonneuve P, Boyle P (1994) Antenatal risk factors for malignant brain tumours in New South Wales children. Int J Cancer 56: $6-10$
McKean-Cowdin R, Pogoda JM, Lijinsky W, Holly EA, Mueller BA, PrestonMartin S (2003) Maternal prenatal exposure to nitrosatable drugs and childhood brain tumours. Int J Epidemiol 32: 211-217

Melean G, Sestini R, Ammannati F, Papi L (2004) Genetic insights into familial tumors of the nervous system. Am J Med Genet C Semin Med Genet 129: $74-84$

Paganini-Hill A, Ross RK (1982) Reliability of recall of drug usage and other health-related information. Am J Epidemiol 116: 114-122

Preston-Martin S, Yu MC, Benton B, Henderson BE (1982) N-Nitroso compounds and childhood brain tumors: a case-control study. Cancer Res 42: $5240-5245$

Ron E, Modan B, Boice Jr JD, Alfandary E, Stovall M, Chetrit A, Katz L (1988) Tumors of the brain and nervous system after radiotherapy in childhood. N Engl J Med 319: 1033 - 1039

Ryan P, Lee MW, North B, McMichael AJ (1992) Risk factors for tumors of the brain and meninges: results from the Adelaide Adult Brain Tumor Study. Int J Cancer 51: 20-27

van der Sanden GA, Schouten LJ, van Dijck JA, van Andel JP, Coebergh J (1998) Incidence of primary central nervous system cancers in South and East Netherlands in 1989-1994. Neuroepidemiology 17: 247-257

WHO (1990) Guidelines for ATC Classification. Oslo: WHO Collaborating Centre for Drug Statistics Methodology - Nordic Council on Medicines Wrensch M, Minn Y, Chew T, Bondy M, Berger MS (2002) Epidemiology of primary brain tumors: current concepts and review of the literature. Neuro-oncology 4: 278-299 